Rev. Elev. Méd. Vét. Poys trop., 1966, 19, 4 (485-494).

\title{
La rickettsiose générale bovine au Sénégal
}

\author{
por M. RIOCHE
}

\begin{abstract}
RÉSUMÉ
L'existence de la rickettsiose générale bovine est signalée au Sénégal. Les travaux déjà effectués dans divers pays sur cette affection sont rappelés. Les symptômes et lẹ́sions sont décrits. La présence de Rickettsia bavis dans le sang et certaines anomalies leucocytaires «presque pathognomaniques 》 parmeitent le diagnostic de la maladie. Le seul traitement efficace consistẹ en injections d'auréomycine par la voie veineuse à la dose de 4 à $5 \mathrm{mg} / \mathrm{kg} / \mathrm{jour}$ pendant 4 à 5 jours. Le rôle d'Amblyoma voriegatum dans la transmission de la maladie est démontré.
\end{abstract}

Alors qu'elle était déjà connue dans plusieurs pays d'Afrique, la rickettsiose générqle bovine à Rickettsia bovis (DONATIEN et LESTOQUARD, 1936) est d'observation récente au Sénégal.

Plușieurs cas de cette maladie ont été en effet identifiés dans la région du Cap Vert au cours de la saison des pluies 1965.

Rickeftsia bovis a été décrite pour la première fois par DONATIEN et LESTOQUARD (1936) en Algérie. Par la suite, G. DE KOCK, VAN HEERDEN, R. DU TOIT et $W$. O. NEITZ la signalent en Afrique du Sud en 1937.

Puis, DONATIEN et LESTOQUARD reprennent leurs travaux et concluent que cette rickettsie, fréquente en Algérie, détermine une maladie bénigne, jamais mortelle (1937, 1938 et 1940).

$\mathrm{Si}$ en Algérie les bovins sont peu sensibles à l'infection à Rickettsia bovis, il n'en n'est pas de même dans d'autres régions. En effet, MALBRANT, BAYROU ef RAPIN en 1939, au Tchad ef au Congo-Brazzaville, puis GIRARD et ROUSSELOT en 1945 au Soudan, décrivent une affection grave déterminée par ce protozoaire. FINELLE en 1958 signale une mortalité de 25 p. 100 en République Centrafricaine.

Dans le cas présent, c'est sous une forme identique, souvent mortelle, que nous l'avons observée au Sénégal, chez des bovins importés.

\section{RÉPARTITION GÉOGRAPHIQUE}

En raison de son observation récente, la répartition des foyers de rickettsiose générale bovine au Sénégal 'n'est pas encore connue. Jusqu'à présent, elle n'est signalée que dans la région du Cap Vert.

Le premier cas clinique est observé à Sangalkam le 17 août 1965. Il s'agit d'une vache zébu de race sahiwal. Cet animal fait partie d'un loł de 12 zébus de même race importés de Tunisie le 13 juillet 1965 en vue d'une amélioration par croisement des races locales.

Cette vache meurt le 15 août.

Puis les cas se succèdent durant les mois d'coût et de septembre.

Sont ainsi identifiés :

- un cas mortel le 21 août,

- un cas le 22 août.

- un cas le 24 août.

- un cas le 31 août,

- trois cas dont un mortel le 13 septembre,

- deux cas le 16 septembre.

Donc, sur les douze animaux formant ce lot, dix contractent la maladie et chaque fois Rickeftsia bovis est identifiée dans les prélèvements effectués. 
Un cas mortel est ensuite observé le 20 septembre, dans un élevage de Sébikotane, sur une vache jersiaise n'ayant jamais eu de contact avec les zébus sahiwal.

Enfin, au mois d'octobre, à Sangalkam, un veau ndama, âgé de 3 mois et maintenus en stabulation depuis sa naissance, est envoyé au pâturage afin de savoir s'il serait atteint de rickettsiose naturelle, ceci dans le but de vérifier si les zébus pakistanais avaient été contaminés sur place. Après quelques temps, Rickettsia bovis est identifiée pendant plusieurs jours dans le sang de l'animal.

\section{Espèces sensibles.}

Nous n'avons observé la maladie naturelle que sur des bovins. DONATIEN et LESTOQUARD (1936) ont démonitré qu'on pouvait transmettre expérimentalement $R$. bovis au mouton qui ne présente qu'une hyperthermie passagère. Ceci a été confirmé par ROUSSELOT (1953).

Nous avons tenté de transmettre la maladie à la chèvre par inoculation intraveineuse de $15 \mathrm{ml}$ de sang d'un bovin infecté. L'animal n'a présenté pendant les deux mois d'observation aucun symptôme et aucune rickettsie n'a pu être mise en évidence dans le sang circulant.

Par ailleurs, des inoculations de sang à la souris (par la voie intra-péritonéale) et au cobaye (par les voies intra-péritonéale et intra-cardiaque) ont été pareillement négatives.

\section{ÉVOLUTION ET SYMPTÔMES}

Les symptômes ef l'évolution de la maladie n'ont pu être éfudiés de façon complète que chez quelques animaux.

\section{a) Evolution.}

Dans la forme suraiguë, deux animaux meurent en 12 heures environ ef la mort est précédée chez l'un par des symptômes nerveux spectaculaires (*). Chez trois animaux, la maladie évolue en 5 à 8 jours (forme aiguë). Un animal guérit en 5 jours après un traitement à l'auréomycine, un autre meurt au bout de

(*) Premier cas elinique (décrit plus loin).
8 jours avec des symptômes de paralysie (**), le dernier, atteint de graves troubles intestinaux, ne résiste que 5 jours (cas de Sébikotane).

\section{b) Symptômes.}

Bien que dans plusieurs cas le traitement ait provoqué une guérison rapide, la présence 'de certains symptômes est constante.

Au début de la maladie, l'animal est triste ef abattu. If se tient debout, immobile, la tête basse, ou couché, indifférent à ce qui l'entoure. Il ne mange pas ef ne rumine plus. La température oscille entre $39^{\circ}$ ef $41^{\circ} \mathrm{C}$. La respiration est accélérée et dyspnéique (à l'auscultation, le murmure vésiculaire est perceptible) et la percussion révèle des zones de matité, en général bilatérales ef localisées aux régions supérieures. La toux, quand elle existe, est sèche et quinteuse. Le rythme cardiaque est accéléré. Dans toutes les formes cliniques, lés excréments sont diarrhéiques.

A côté de ces symptômes qui sont constants, existent assez souvent des signes nerveux. ils peuvent être relativement bénins et disparaître après la guérison : paralysie du larynx (cornage) ou signes d'hyper-excitabilité.

Cependant, chez certains animaux, ces derniers peuvent être graves. Deux cas ont été observés :

Premier cas : taureau sahiwal (évolution suraiguë en 12 heures).

Phases d'excitation ef de somnolence. Crises épileptiformes avec chute sur le sol, yeux révulsés, salivation ef mouvements de galop. Après la crise, état d'hébétude puis relever pénible suivi d'une période de somnolence avec tête appuyée contre le mur. ef tremblements musculaires généralisés. La mort survient au cours d'une crise épileptiforme.

Deuxième cas :'vache zébu sahiwal (évolution aiguë en 8 jours).

Dès le début de la maladie, lever et déplacements difficiles; membres postérieurs raidis (sans doute par gêne articulaire) donnant à la démarche une allure titubante. L'animal bute fréquemment. Au bout de deux jours, decubitus sternal, station debout pénible. Le cinquième jour, decubitus latéral, station debout imposșible.

(**) Deuxième cas clinique (décrit plus loin). 
Paralysie apparente avec de courts moments d'excitation (esquisse de mouvements de galop). Insensibilité cutanée des membres et de la croupe. Disparition du réflexe pupillaire. Opisthotonos permanent. Dès le quatrième jour. signe de l'oreille (GIRARD ef ROUSSELOT, 1945). Température oscillant entre 38,5 et $39,5^{\circ} \mathrm{C}$. L'évalution se termine par la mort de l'animal.

\section{LÉSIONS}

Les muscles peauciers et le conjonctif souscutané sont le siège d'une congestion intense qui peut s'étendre à toutes les séreuses. Hydropéricarde constant, sérofibrineux, parfois hémorragique. Epanchements citrins dans les cavités pleurales et péritonéale.

Lésions hémorragiques constantes dont les plus caractéristiques siègent sur le myocarde et les troncs artèriels, Ce sont des pétéchies et suffusions șanguines (myocarde) et des placards hémorragiques, le plus souvent à la base de l'artère pulmonaire ou le long de l'corte thoracique. Lésions hémorragiques fréquentes au niveau de la rate, du péricarde, des poumons et de la plèvre.

Congestion active ef cedème aigu du poumon. avec parfois adème périlobulaire important donnant un aspect en mosaïque différent cependant de celui observé dans la péripneumonie bovine.

\section{Autres lésions :}

- foie congestionné ef friable,

- rate réactionnelle (granuleuse au toucher).

- ganglions lymphatiques toujours hypertrophiés et succulents, parfois congestionnés.

- congestion rénale.

\section{Lésions nerveuses :}

Congestion plus ou moins intense des méninges. Chez une vache sahiwal $\left(2^{\mathrm{e}}\right.$ cas clinique décrit), congestion intense de la pie-mère qui est très épaissie et porte sur sa face externe de très nombreux nodules blanchâtres de la grosseur d'une tête d'épingle, disséminés sur toute la méninge; mais particulièrement abondants au niveau du bulbe, de la face inférieure du tronc cérébral et de la moelle épinière. Pétéchies et placiards hémorragiques très nombreux sur la pie-mère de la moelle lombosacrée et de la queue de cheval (photos 1, 2, 3 et 4).

\section{AGENT CAUSAL}

L'examen des frottis de sang périphérique ef des frottis par apposition de rate, poumon, rein, foie, ganglions lymphatiques, colorés par la méthode de May-Grunwald-Giemsa, permet de mettre en évidence Rickettsio bovis soit dans les monocytes, soit dans les macrophages (Photos 5,6 et 7 ).

\section{Sang périphérique,}

Toutes les inclusions observées présentent la morphologie déjà décrite par de nombreux auteurs (DONATIEN et LESTOQUARD, 1936, 1937, 1940), (GIRARD et ROUSSELOT, 1945), (ROUSSELOT, 1953).

\section{a) Corps initial.}

Taille comprise entre 2 et $8 \mu$. Sa coloration varie du lilas clair au violet foncé. Lorsqu'il est gros, le corps initial paraît souvent formé de plusieurs masses sombres noyées dans une gangue de couleur plus claıre. II refoule souvent le noyau en donnant l'ımpression d'être logé dans une encoche de celui-ci. Son contour est en général régulier ef arrondi mais peut être irrégulier.

En principe, on ne trouve qu'une seule inclusion par cellule mais il n'est pas rare d'en observer plusieurs, de taille variable. Les corps initiaux sont souvent situés dans une vacuole et paraissent de ce fait entourés d'un halo clair.

\section{b) Morula.}

C'est un corps initial en voie de fragmentation qui se colore par le May-Grunwald-Giemsa en rose litas ou en gris mauve. Elle est ronde et d'aspect granuleux. Comme le corps initial, elle est souvent située dans une vacuole du cytoplasme. Sa taille varie dans les mêmes proportions que celle du corps initial et comme lui, elle peut refouler la membrane nucléaire.

\section{c) Corps élémentaire.}

Ils sont de petite taille $(0,2$ d̀ $0,8 \mu)$ et en général de coloration lilas. Leur situation à la limite de la visibilité les rend difficiles à étudier 


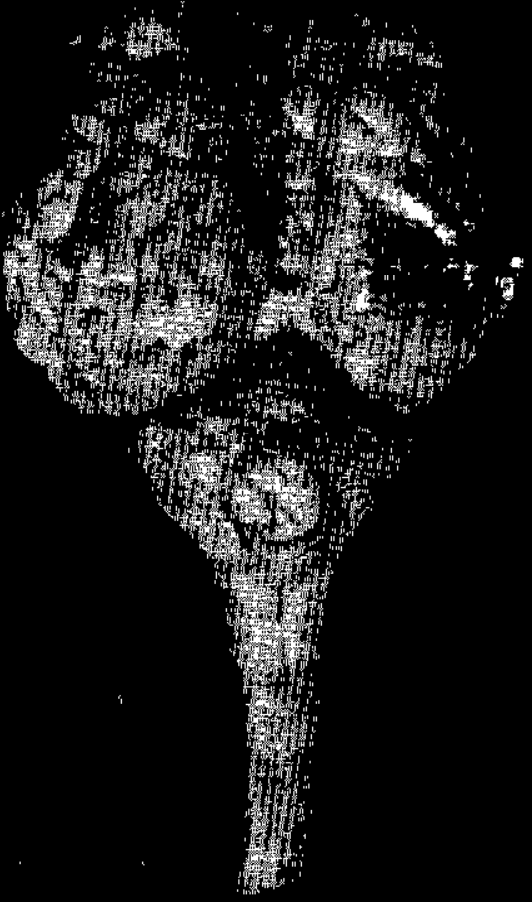

Photo no 1. - Rickettsiose bovine à Rickettsia bovis. Cas clinique $n^{0} 2$. Lésions nodulaires de la pie-mère

Photo no 2. - Rickettsiose bovine à Rickettsio bovis. Lésions nodulaires et lésions hémorragiques de la pie-mère : moelle lombo-sacrée. 


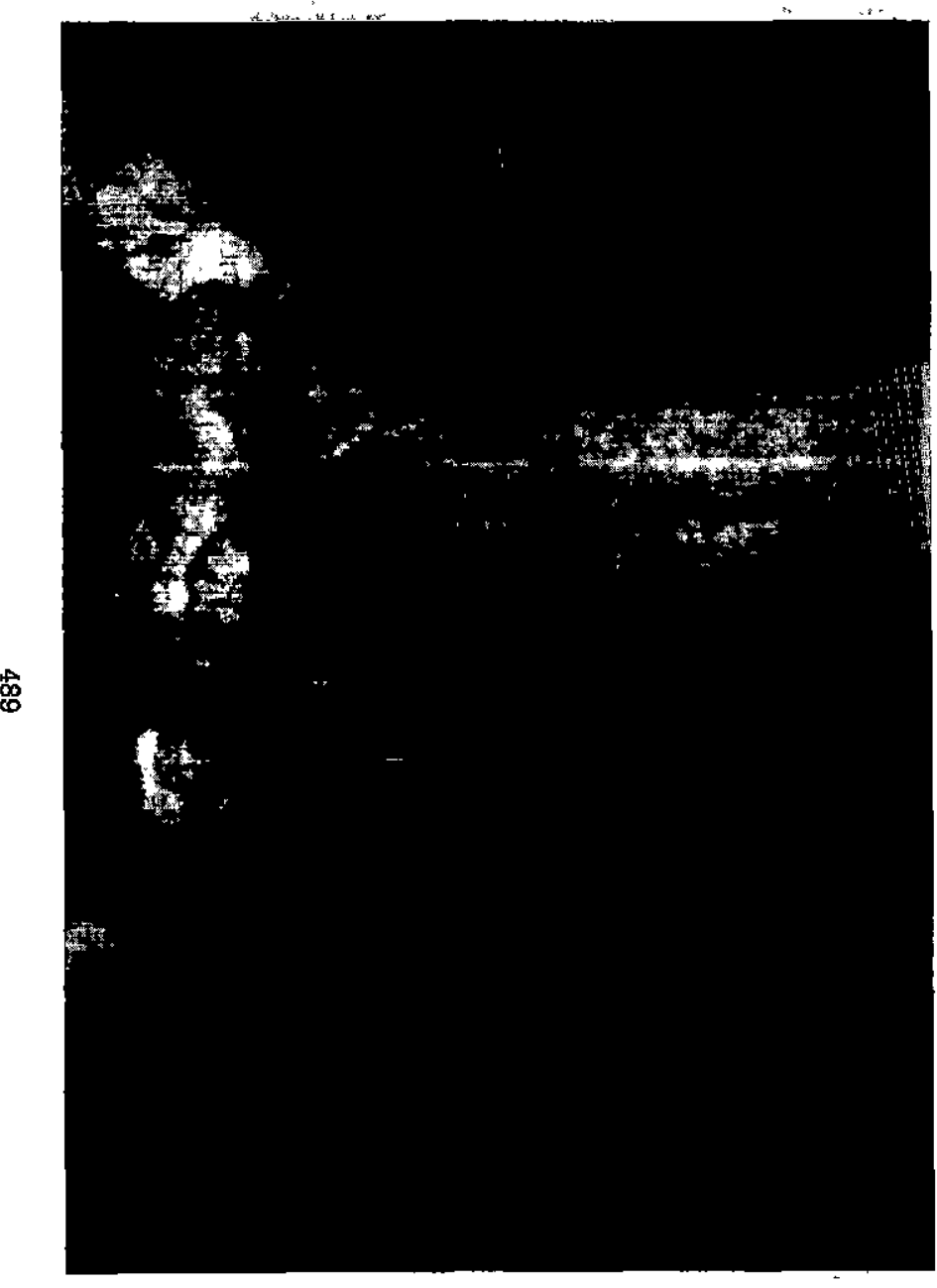

Photo $n^{0 \cdot 3 .}$ - Rickettsıse bovine à Rickefisio bovis Lésions nodulaires de la pie-mère : cervelet ef bulbe.

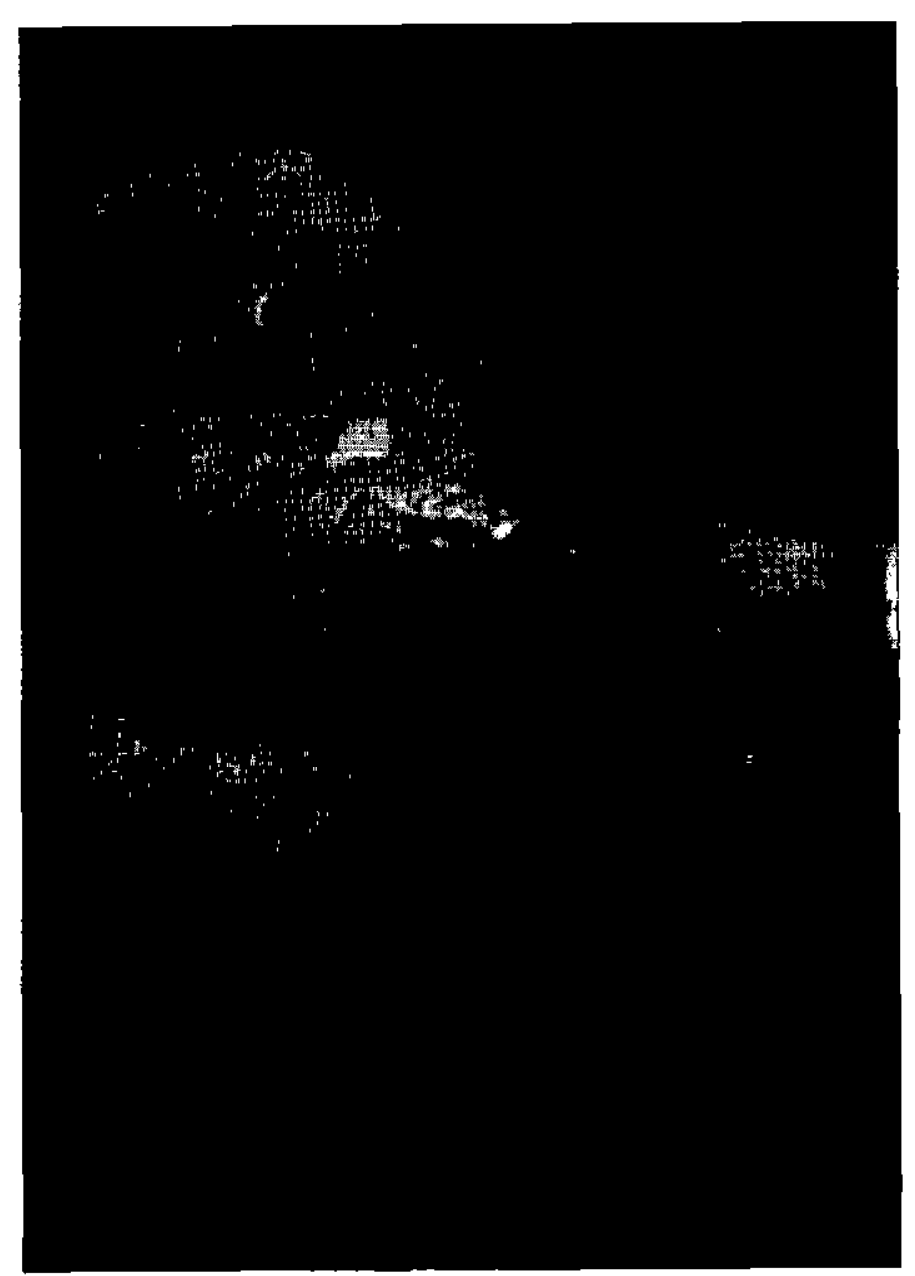

Photo n० 4. - Rickettsiose bovine à Rickettsia bovis. Lésions nodulaires de la pie-mère : face inférieure du tronc cérébral. 


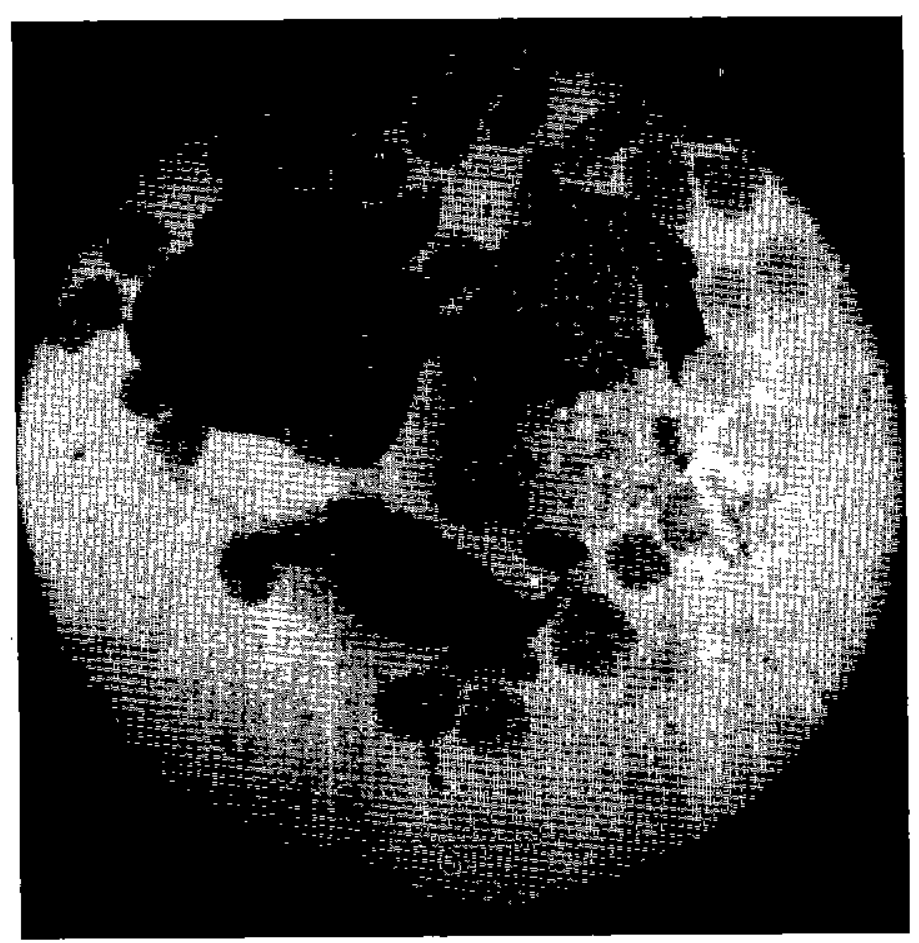

Photo $n^{0}$ 5. - Rickettsia bovis : sang périphérique.

Au centre, manocyte contenant un amas de corps élémentaires.

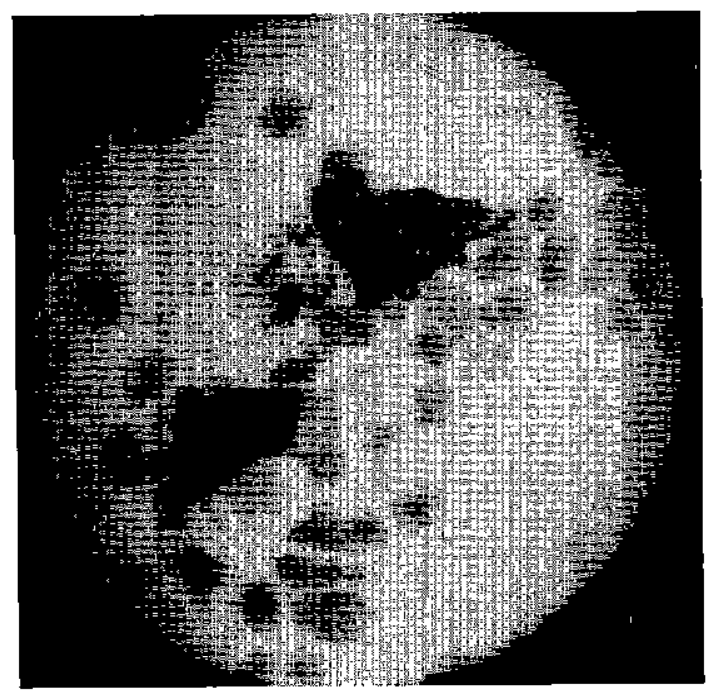

$$
10 \mu
$$

Photo $n^{\circ}$ 6., - Rickettsia bovis : sang périphérique. Monocyte contenant des corps initiaux et quelques corps élémentaires (eh haut).

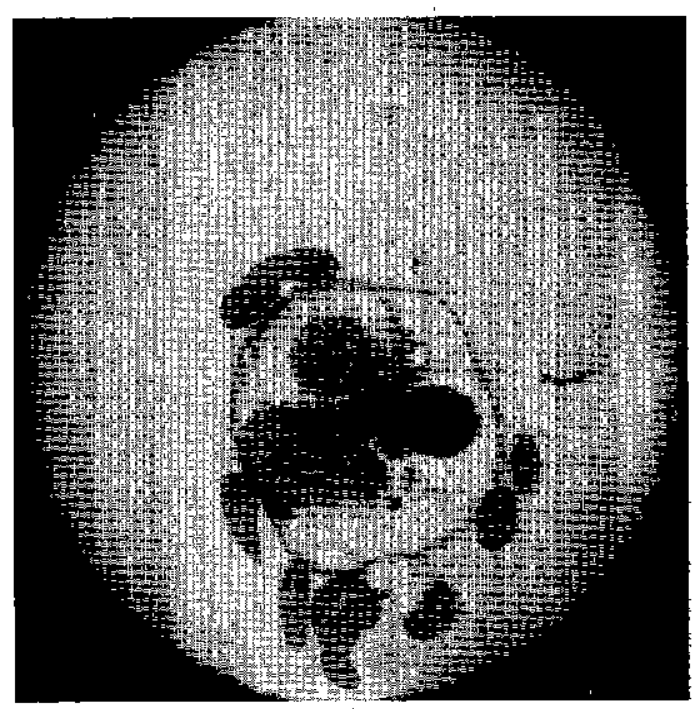

Clichés GRÉTILLAT (S.) et RIOCHE (M.)

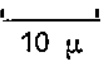

Photo no 7. - Rickettsia bovis : frottis de poumon. Macrophage contenont quatre corps initiaux (à droite du noyau) et une morula (au-dessus du noyau). 
sauf lorsqu'ils sont groupés en amas ou situés isolément dans de petites vacuoles. Leur morphologie est coccoide ou cocco-bacillaire.

La présence de Rickettsı bovis dans le sang s'accompagne de lésions sanguines.

Tous les auteurs qui ont étudié les rickettsioses des monocytes mentionnent l'existence d'une monocytose. La proportion des monocytes est augmentée ef le taux de ces cellules peut dépasser 20 p. 100. ROUSSELOT (1953) insiste sur la valeur diagnostique de cette augmentation; mais la monocytose seule ne suffit pas. En effet, la formule leucocytaire des bovins varie sensiblement d'un jour à l'autre et l'on peut parfois observer une monocytose moyenne en l'absence de ricketisiose.

Dans la rickettsiose générale bovine, la monocytose est accompagnée d'un ensemble d'outres modifications qui constituent avec elle un tableau presque pathognomonique.

En effet, outre qu'il y a augmentation de leur pourcentage, la plupart des monocytes ont un cytoplasme fortement vacuolaire (dans ces vacuoles on observe souvent des masses grisâtres à contours indistincts, déjà décrites par DONATIEN ef LESTOQUARD, 1936, 1937 ; on peut aussi y voir des corps élémentaires). Souvent, le noyau, bien qu'il conserve sa structure habituelle, a des contours plus ou moins tourmentés ou un aspect « déchiqueté 》.

On trouve aussi des cellules ou des formes cellulaires dont la présence dans le sang circulant est anormale : nombreuses cellules jeunes, macrophages, cellules en mitose.

Enfin, il y a raréfaction et même souvent disparition des éosinophiles, anomalie sıgnalée par DONATIEN et LESTOQUARD (1936) dans la rickettsiose canine à Rickettsia canis. Il semble d'ailleurs qu'il s'agisse d'une éosinopénie réelle car les éosinophiles sont rares ou absents dans les frottis d'organes hématopoiétiques des animaux atteints.

II est important d'insister sur ces modifications de l'image sanguine carce sont elles qu'on remarque en premier lieu à l'examen d'un frottis de sang et que ces lésions sont toujours très visibles alors que, même dans les formes graves, les rickettsies peuvent être rares ef réclamer un examen minutieux. L'observation de ces anomalies permettra souvent de poser un diagnostic précoce, même en l'absence de Rickettsio.

\section{Organes divers.}

Si les prélèvements sont faits dès la mort de l'animal, il est possible de faire des frottis de sang et d'y identifier le parasite. Dans le cas contraire, et de toute façon, on trouve les rickettsies dans les frottis des divers organes. Les plus riches sont les poumons, puis les reins, le foie et la rate. Les Ricksettsia se trouvent soit dans les monocytes du sang de ces organes, soit dans les macrophages.

Il faut insister sur le fait qu'on ne trouve jamais Rickettsia bovis dans les cellules réticulo-endothéliales de la, tunique interne des vaisseaux sanguins.

\section{DIAGNOSTIC}

Les symptômes décrits ne permettent pas de poser le diagnostic avec certitude mais seulement de suspecter la maladie. Seul l'examen des frottis de sang permet de le confirmer. Il en est de même pour le diagnostic nécropsique.

\section{DIAGNOSTIC DIFFERENTIEL}

II doit se faire avec la heart-water. En effet, les symptômes et lésions de la rickettsiose générale bovine sont assez voisins de ceux de la heart-water. Cependant, à l'autopsie, l'œdème périlobulaire observé ne semble pas avoir été décrit dans la heart-water. II en est de même de la congestion du tissu conjonctif sous-cutané ef des muscles peauciers car, selon CURASSON (1943), elle n'existerait pas dans la heart-water où, au contraire «... on a souvent l'impression d'un anımal saigné pour la boucherie».

Toutefois, le diagnostic ne peut être posé avec certitude que par l'examen des prélèvements effectués et la mise en évidence de l'agent causal.

Dans la rickettsiose générale bovine, Rickettsia bovis est identifiée dans les monocytes du sang circulant ou des organes; elle ne l'est jamais dans les cellules de l'endothelium vasculaire.

A l'inverse, Cowdria (Rickettsia) ruminantium s'observe dans les cellules réticulo-endothéliales de la tunique interne des vaisseaux et jamais dans le sang circulant.

Non seulement nous avons identifié Rickettsia bovis dans tous les cas, mais nous avons aussi recherché systématiquement à mettre en évidence 
Cowdria ruminantium sur le cadaure. L'examen des frottis effectués (endothélium de la veine jugulaire, frottis de plexus choroïde ef calques de cerveau fraîchement prélevés) ont été négatIfs. Ceci a permis aussi d'infirmer l'existence possible d'une infection mixte à Rickettsia bovis et à Cowdria ruminontium.

\section{PRONOSTIC}

Le pronostic de l'affection est sérieux puisque sur 14 cas identifiés, 4 ont été mortels.

Par ailleurs, la morbidité est élevée, au moins chez le bétail importé, puisque sur 12 zébus importés, 10 contractent la maladie parmi lesquels 3 meurent ( 2 de forme suraiguë et 1 de forme aigue) et 7 (dont 5 présentent une forme grave d'emblée) guérissent en 3 d̀ 7 jours après un traitement par l'auréomycine.

\section{MODE DE CONTAMINATION}

Pour expliquer l'apparition de cette maladie non encore signalée au Sénégal, on ne peut retenir l'hypothèse selon laquelle les zébus pakistançis, déjà prémunis avant leur importation, avaient fait un accès parasitaire déclenché par les agressions dues au voyage et à l'acclimatement.

\section{En effet :}

a) Rickettsia bovis n'a jamais été signalée en Tunisie, à notre connaissance.

b) Si la crise d'acclimatement avait provoqué un tel accès parasitaire, tous les animaux auraient été malades en même temps, à peu de jours d'intervalle et peu après leur arrivée au Sénégal. Or ici, non seulement le premier cas est apparu plus d'un mois après l'importation des zébus (et ceux-ci étaient alors acclimatés ainsi qu'en témoignait leur bon état général) mais encore la répartition dans le temps des cas de ricketisiose s'étale sur une période supérieure d̀ un mois.

L'origine de cefte affection ne peut donc être qu'autochtone. Les faits suivants le confirment.

Chez les zébus sahiwal normalement maintenus en stabulation libre (l'enclos dans lequel ils évoluent étant dépourvu d'lxodes) nous constatons que les deux premiers cas apparaissent après une sortie malencontreuse au pâturage d'où les animaux reviennent parasités par des tiques (surtout des Amblyomma voriegatum). Par la suite, bien qu'ils ne soient plus sortis et qu'ils soient soumis régulièrement à un traitement ixodicide, les animaux hébergent quelques tiques vraisemblablement introduites dans l'étable avec le fourrage vert dont on les nourrit. Tous les animaux atteints sont parasités par ces arthropodes.

A la suite de ces observations, nous constatons la contamination naturelle au pâturage d'un veau ndama à Sangalkam (cf. Répartition géographique). Une semaine après sa mise au pâturage, it présente une hyperthermie pendant 48 heures $(39,9$ o C le 1er jour, $39,6 \circ \mathrm{C}$ le second) sans autre symptôme morbide. Dès le premier jour d'hyperthermie, des examens quotidiens d'étalements de sang sónt pratiqués et à partir du ge jour, Rickettsia bovis est identifiée dons ces prélèvements. Les rickettsies persịstent pendant 6 jours puis disparaissent. Ce veau fait donc une forme inapparente de la maladie qui serait passée inaperçue s'il n'avait été soumis à une surveillance particulière. Ce fait prouve cependant la réalité de la contamination sur place.

D'autre part, un broyat de tiques est inoculé à deux bovins de race locale. Réalisé avec des Amblyomma variegatum récoltés sur des bovins de race locale, pâturant dans la région de Thiès, ce broyat est mis en suspension dans du milieu HBSS (Hank's balanced salted solution) pour culture de tissus ef inoculé (sans antibiatique) sous la peau des deux animaux. Respectivement, 16 et 29 jours après l'inoculation, Rickettsia bovis est idenfifiée dans les frottis de sang de ces bovins, persiste quelques jours puis disparaît. La présence des rickettsies s'accompagne des modifications sanguines déjà décrites. Ces animaux font une forme inapparente de la maladie car en dehors d'une légère baisse d'état, ils ne présentent aucun symptôme morbide.

Cette expérimentation permet de démontrer la transmission de Rickettsia bovis par Amblyommo variegatum ef d'établir que la région de Thiès est un foyer de rickettsiose générale bovine.

Il faut donc conclure à l'existence de la rickettsiose générale bovine au Sénégal et' que ceite 
maladie se montre particulièrement grave chez les zébus pakistanais, animaux neufs ef peutêtre particulièrement sensibles à cette affection.

\section{TRANSMISSION TRANSPLACENTAIRE}

Une des vaches mortes de rickettsiose étant en gestation, nous avons fait l'autopsie du fœetus et examiné des frottis de sang et des calques d'organes. Le fœitus, âgé de trois mols, était indemne de lésions et la recherche des rickettsies est restée négative. Cependant, l'étude de ce cas unique ne permet pas de tirer de conclusion sur la transmission ou la non-transmission de cette rickeftsie par la voie placentaire.

\section{TRAITEMENT}

Parmi les traitements essayés, l'utilisation de l'association pénicilline-streptomycine (3 millions $\mathrm{U} .1 .-3 \mathrm{~g} /$ /jour) ou de la tétracycline en injection intramusculaire s'est toujours soldée par des écheas.

Seule l'auréomycine par la voie intraveineuse, è la dose journalière de 4 à $5 \mathrm{mg} / \mathrm{kg}$ ef pendant 4 à 5 jours, a permis d'assurer une guérison rapide des animaux. Mais son efficacité semble illusoire dans les formes suraiguës.

A ce-traitement spécifique est ajouté une thérapeutique symptomatique (tonicardiaque).

\section{PROPHYLAXIE}

La seule prophylaxie actuellement possible est la lutie contre les tiques. II est nécessaire de traiter les animaux à l'aide d'un produit acaricide au moins une fois par semaine et, en plus de ce traitement, de maintenir les bovins récemment importés en stabulation permanente.

\section{CONCLUSION}

C'est la première fois, à notre connaissance, que la rickettsiose générale bovine est signalée au Sénégal. Cette observation porte à deux, le nombre de rickettsioses des monocytes identifiées dans ce pays, puisque, en 1941; CURASSON y signale l'existence de la rickettsiose générale ovine à Rickettsia ovina (LESTOQUARD ef DONATIEN, 1936).

Dans ce pays, comme dans toutes les régions tropicales, Rickettsia bovis est réellement pathogène alors que DONATIEN ef LESTOQUARD, 1940, constatent qu'en Algérie, elle ne provoque que des maladies bénignes. CURASSON (1942) avaił fait la même remarque au sujet du rôle pathogène de Rickettsia ovina. Aussi nous pensons que, suivant ROUSSELOT (1953), if faut «... accorder à ces affections décrites comme inapparentes en Algérie une pathogénicité spéciale lorsqu'elles interviennent en pays tropical ».

Dans les quelques cas que nous avons observés, c'est surtout pour le bétail récemment importé que cette rickettsiose a été très grave. Nous ne disposons pas encore d'observations cliniques concernant la maladie naturelle sévissant sur le bétail de race locale pour pouvoir juger de sa gravité éventuelle pour le cheptel sénégalais.

Institut d'Elevage ef de Médecine
vétérınaire des Pays Tropicaux
Maisons-Alfort
Laboratoire national de l'Elevage
ef de Recherches vétérinaires
Dakar-Hann

Institut d'Elevage ef de Médecine Maisons-Alfort Dakar-Hann

\section{SUMMARY}

\section{Bovine Rickettsiosis in Senegal}

Bovine Rickettsiosis has been recorded in Senegal. The work which has been carried out on this disease in some various countries is reported. The clinical signs and lesions are described. Diagnose of the disease is made through the evidence of $R$. bavis in the blood of infected animals and the presence in it of some abnormal leucocytes which are nearly characteristic of the disease. The only effecive treaiment consists in Aureomycine. Intraveinous injections at the dose of 4 to $5 \mathrm{mg} / \mathrm{kg} /$ day during 4 or 5 days.

The part played by Amblyomma variegatum in the iransmission of the disease has been established. 


\title{
RESUMEN
}

La Rickettsiosis general bovina en Senegal

\begin{abstract}
Se nota la existencia de la Rickettsiosis general bovina en Senegal. Se pasan en revista los trabajos ya efectuados en varios paises concerniendo a esta enfermedad. Las sintomas y las lesiones se describen. La presencia de Rickettsia bovis en la sangre y algunas anomalías leucocitarias «casi patognomónicas» permiten diagnosticar la enfermedad. Las inyecciones de aureomicina por via venosa en la dosis de 4 a $5 \mathrm{mg} / \mathrm{kg} /$ dia durante 4 o 5 dias son el solo tratamıento activo. Se demuestra el papel de Amblyomma variegatum en la transmisión de la enfermedad.
\end{abstract}

\section{BIBLIOGRAPHIE}

CURASSON (G.). - La rickettsiose générale ovine au Sénégal. Première note : La maladie expérimentale. Bull. Serv. Zootechn. ef des Epiz., 1941, 4 (1), 34-36.

CURASSON (G.). - La rickettsiose générale ovine au Sénégal. Bull. Serv. Zootechn. ef des Epiz,, 1942, 5 (1) : 3-8.

CURASSON (G.). - Protozoologie vétérinaire et comparée T. III, Vigof Frères, 1943, $493 \mathrm{p}$.

DONATIEN (A.) et LESTOQUARD (F.). Recherches sur Rickeftsia canis. Comparaison avec Rickettsia conori. Bull. Soc. Path. exot., 1936, 39 (10) : 1052-56.

DONATIEN (A.) et LESTOQUARD (F.). Rickettsia bovis nouvelle espèce pathogène pour le bouf. Bull. Soc. Path. exot., 1936, 39 (10), 1057-61.

DONATIEN (A.) ef LESTOQUARD (F.). Etat actuel des connaissances sur les rickettsioses animales. Arch. Inst. Pasteur Algérie, 1937, 15 (2) : 142-87.

DONATIEN (A.) ef LESTOQUARD (F.). Particularités des Rickettsia des monocytes. Bull. Acad. vét., 1937, (10), 183-87.

DONATIEN (A.) et LESTOQUARD (F.). Les rickettsioses animales. Rev. Méd. vét., 1938, 2, 121-39.
DONATIEN (A.) et LESTOQUARD (F.). Rickettsiose bovine algérienne à Rickettsia bovis. Bull. Soc. Path. exot., 1940 (3), 245-48. FINELLE (P.). - Note clinique Rickettsiose à Rickettsia bovis en Oubangui-Chari. Rev. Elev. Méd. vét. Pays trop., 1958, 291-92.

GIRARD (H.) et ROUSSELOT (R.). - La rickettsiose à Rickettsia bovis, protiste pathogène au Soudan français. Bull. Soc. Path. exot., 1945 (3-4) : 64-77.

GIRARD ( $H_{\text {. }}$ ) et ROUSSELOT $\left(R_{0}\right)$. - - Les rickettsioses animales' au Soudan. Encycl. vét. périod., 1947 (11-12), 403-18.

$\operatorname{KOCK}(G$. de), VAN HEERDEN (C. J.), DU TOIT (R.) et NEITZ (W. O.). - Theileriose bovine en Afrique du. Sud avec référence spéciale sur Th. mufans (Bovine theileriasis in South Africa with special reference to Th. mufons). Onderstepoort J. vet. Res., 1937, 8, (1-2), 9-128.

LESTOQUARD (F.) et DONATIEN (A.). Sur une nouvelle Rickettsia du mouton. Bull. Soc. Path. exot., 1936 (2), 105-8.

MALBRANT (R.), BAYROU (M.) ef RAPIN (P.). - Protozooses sanguines des animaux domestiques en A. E. F. Bull. Soc. Path. exot., 1939, 32 (10), 953-60.

ROUSSELOT (R.). - Notes de parasitologie tropicale. Parasites du sang des animaux (tome 1), Vigot Frères, 1953, $152 \mathrm{p}$. 